GANIT J. Bangladesh Math. Soc. (ISSN 1606-3694) 37 (2017) 1-14

\title{
EXACT TRAVELING WAVE SOLUTIONS TO BENNEY- LUKE EQUATION
}

\author{
Zahidul Islam $^{1, *}$, Mohammad Mobarak Hossain ${ }^{2}$ Md. Abu Naim Sheikh ${ }^{3}$ \\ ${ }^{1,3}$ Department of Mathematics, Dhaka University of Engineering \& Technology, \\ Gazipur, Bangladesh. \\ ${ }^{2}$ Department of Mathematics, Hamdard University Bangladesh, Bangladesh \\ ${ }^{*}$ Corresponding author, email: zahiduhsc@gmail.com
}

Received 28.04.2016 Accepted 25.05.2017

\begin{abstract}
By using the improved $\left(G^{\prime} / G\right)$-expansion method, we obtained some travelling wave solutions of well-known nonlinear Sobolev type partial differential equations, namely, the Benney-Luke equation. We show that the improved $\left(G^{\prime} / G\right)$-expansion method is a useful, reliable, and concise method to solve these types of equations.
\end{abstract}

Keywords: Traveling Wave, Benney-Luke Equation, Sobolev type PDEs

\section{Introduction}

The nonlinear evolution equations (NLEEs) containing the term with spatial derivatives on the highest order time derivative is refer as Sobolev type equation [1]. Equations of Sobolev type characterized by having mixed time and space derivatives appearing in the highest-order terms of the equation were studied by Sobolev [2] and this type of equation can be used to describe numerous physical phenomena [3-7].

The nonlinear evolution equations (NLEEs) are very much significant due to its wide-ranging applications. In modern science nonlinear phenomena are one of the most notable fields of research and occur in numerous branches of science and engineering, such as, plasma physics, fluid mechanics, gas dynamics, elasticity, relativity, chemical reactions, ecology, optical fiber, solid state physics, biomechanics, etc., all are fundamentally governed by nonlinear equations. NLEEs are frequently used to demonstrate the motion of isolated waves.

Since the appearance of solitary wave in natural sciences is intensifying day by day, it is important to seek for exact traveling wave solutions to NLEEs. The exact solutions to NLEEs help us to provide information about the structure of complex physical phenomena. Therefore, investigation of exact traveling wave solutions to NLEEs turns into an essential mission in the study of nonlinear physical phenomena. It is notable to observe that there is no unique method to solve all kind of NLEEs. For this reason, a lot of methods have been established, such as, the Jacobi elliptic function method [8], the homotopy perturbation method [9], the variational method [10], the Adomian Decomposition Method [11], the modified simple equation method [12,13], the Exp- 
function method[14], the $\left(G^{\prime} / G\right)$-expansion method [15-17], the multiple exp-function method [18], the tanh method [19], the extended tanh method [20], the sine-cosine function method [21], the $\exp (-\Phi(\xi))$-expansion method [22, 23], various important analytical method [24-30] and so on.

Benney-Luke is very important Sobolev type equation and a formally valid approximation for describing two-way water wave propagation in presence of surface tension studied for a very long time. For its impotency, many researchers studied about the equation and have many research article of equation in the literature. Wang et. al. [24] studied the Cauchy problem for the generalized Benney-Luke equations. Gonz'alez [25] discussed about the Cauchy problem for Benney-Luke and generalized Benney-Luke equations. Quintero [26] made a remark on the Cauchy problem for the generalized Benney-Luke equation. Quintero and Grajales [27] investigated instability of solitary waves for the generalized Benney-Luke equation. Most recently Akter and Akbar [31] studied the Benney-Luke equation and the Phi-4 equations by using modified simple equation method to find exact solutions. Gozukizil and Akcagil [32] also studied the Benney-Luke equation to find some exact traveling wave solutions.

To the best of our knowledge the improved $\left(G^{\prime} / G\right)$ expansion method with both positive/negative value of suffices in the trial solution has not been applied to the above mentioned equation in the previous literature. In this paper, we investigate the traveling wave solutions of the Benney-Luke equation (1) with auxiliary equation $G^{\prime \prime}+\lambda G^{\prime}+\mu G=0$ and consider both the positive and negative value of suffices in the trial solutions.

\section{Description of the improved $\left(G^{\prime} / G\right)$-expansion method}

Suppose that we have a NLEE for $U(x, t)$ in the form

$$
P\left(U, U_{x}, U_{t}, U_{x x}, U_{x t}, U_{t t}, \ldots \ldots\right)=0
$$

where $\mathrm{P}$ is a polynomial in its arguments, which includes nonlinear terms and the highest order derivatives. The transformation

$$
U(x, t)=u(\xi), \xi=x-w t
$$

permits us in reducing Eq.(2) to an ODE for $u=u(\xi)$

$$
P\left(u, u^{\prime}, u^{\prime \prime}, \cdots \cdots \cdots\right)=0
$$

Step 2. Suppose that the solution of ODE (3) can be uttered by a polynomial in $\left(G^{\prime} / G\right)$ as follows

$$
u=\sum_{i=0}^{m} A_{i}\left(G^{\prime}(\xi) / G(\xi)\right)^{i}+\sum_{i=1}^{m} B_{i}\left(G(\xi) / G^{\prime}(\xi)\right)^{i}
$$

where $G(\xi)$ satisfies the ODE in

$$
G^{\prime \prime}(\xi)+\lambda G^{\prime}(\xi)+\mu G(\xi)=0,
$$

then the solutions of ODE (5) are

When $\lambda^{2}-4 \mu>0$, then 


$$
G^{\prime} / G=\frac{\sqrt{\lambda^{2}-4 \mu}}{2}\left(\frac{C_{1} \sinh \left(\frac{\sqrt{\lambda^{2}-4 \mu}}{2} \xi\right)+C_{2} \cosh \left(\frac{\sqrt{\lambda^{2}-4 \mu}}{2} \xi\right)}{C_{1} \cosh \left(\frac{\sqrt{\lambda^{2}-4 \mu}}{2} \xi\right)+C_{2} \sinh \left(\frac{\sqrt{\lambda^{2}-4 \mu}}{2} \xi\right)}\right)-\frac{\lambda}{2}
$$

When $\lambda^{2}-4 \mu<0$, then

$$
G^{\prime} / G=\frac{\sqrt{4 \mu-\lambda^{2}}}{2}\left(\frac{-C_{1} \sin \left(\frac{\sqrt{4 \mu-\lambda^{2}}}{2} \xi\right)+C_{2} \cos \left(\frac{\sqrt{4 \mu-\lambda^{2}}}{2} \xi\right)}{C_{1} \cos \left(\frac{\sqrt{4 \mu-\lambda^{2}}}{2} \xi\right)+C_{2} \sin \left(\frac{\sqrt{4 \mu-\lambda^{2}}}{2} \xi\right)}\right)-\frac{\lambda}{2}
$$

When $\lambda^{2}-4 \mu=0, \mu=\lambda=0$, then $G^{\prime} / G=\frac{C_{1}}{C_{1} \xi+C_{2}}$

$A_{i}, B_{i}, w, \lambda, \mu ; i=0, \cdots \cdots, m$ are constants to be determined later, in which $A_{m}$ and $B_{m}$ are not both zero at the same time. Solitons are the outcome of a delicate balance between nonlinearity and dispersion and so the positive integer $m$ can be resolute by considering the homogeneous balance between the highest order derivatives and nonlinear terms arising in ODE (3). By substituting (4) into Eq.(3) and using the ODE (5), collecting all terms with the same order of $\left(G^{\prime} / G\right)^{i} ; i=0, \cdots \cdots, m$ together, and setting them to zero, yields a system of algebraic equations for $A_{i}, B_{i}, w, \lambda, \mu ; i=0, \cdots \cdots, m$ and $\mu$.

Now, solving the algebraic equations for $A_{i}, B_{i}, w, \lambda, \mu ; i=0, \cdots \cdots, m$ and $\mu$ and putting them in the general solutions of ODE (5), we obtain the general solutions of Eq.(1).

\section{Traveling Wave Solution of Benney-Luke Equation}

In this section, we will make use of the improved $\left(G^{\prime} / G\right)$-expansion method to find the exact explicit, solitary and periodic wave solutions to the Benney-Luke equation. Let us consider the Benney-Luke equation in the form

$$
u_{t t}-u_{x x}+\alpha u_{x x x x}-\beta u_{x x t t}+u_{t} u_{x x}+2 u_{x} u_{x t}=0
$$

where $\alpha$ and $\beta$ are positive number such that $\alpha-\beta=\sigma-1 / 3$ is a Sobolev type equation and studied for a very long time. The dimensionless parameter $\sigma$ is named the Bond number, which captures the effects of surface tension and gravity force and is a formally valid approximation for describing two-way water wave propagation in presence of surface tension [24-26, 31-32].

Now making use of the traveling wave variable $\xi=x-w t$ into (1) we amend Eq. (1) into the ODE:

$$
\left(w^{2}-1\right) u^{\prime \prime}+\left(\alpha-\beta w^{2}\right) u^{i v}-3 w u^{\prime} u^{\prime \prime}=0
$$

Integrating Eq. (10) once with respect to $\xi$ and setting the integration constant equal to zero yields 


$$
\left(w^{2}-1\right) u^{\prime}+\left(\alpha-\beta w^{2}\right) u^{\prime \prime \prime}-\frac{3}{2} w\left(u^{\prime}\right)^{2}=0
$$

Balance the highest order derivate term $u^{\prime \prime \prime}$ and the highest nonlinear terms $u^{\prime 2}$ in equation (11), we get $m=1$, so the method admits the solution of the Eq.(3) in the form

$$
u(\xi)=A_{0}+A_{1}\left(G^{\prime} / G\right)+B_{1}\left(G / G^{\prime}\right)
$$

where $u(x, t)=u(\xi), \xi=x-w t$ and $A_{1}$ and $B_{1}$ are not both zero at the same time.

Differentiating $\mathrm{Eq}(12)$ and using the condition $\mathrm{Eq}(5)$, we reach the following equations

$$
\begin{aligned}
& u^{\prime}(\xi)=-A_{1}\left(G^{\prime} / G\right)^{2}-A_{1} \lambda\left(G / G^{\prime}\right)+B_{1}-A_{1} \mu+B_{1} \lambda\left(G^{\prime} / G\right)^{-1}+B_{1} \mu\left(G^{\prime} / G\right)^{-2} \\
& u^{\prime \prime}(\xi)=-A_{1}\left(G^{\prime} / G\right)^{3}+3 A_{1} \lambda\left(G^{\prime} / G\right)^{2}+A_{1}\left(\lambda^{2}+2 \mu\right)\left(G^{\prime} / G\right)+A_{1} \lambda \mu+B_{1} \lambda \\
& +B_{1}\left(\lambda^{2}+2 \mu\right)\left(G / G^{\prime}\right)^{-1}+3 B_{1} \lambda \mu\left(G^{\prime} / G\right)^{-2}+2 B_{1} \mu^{2}\left(G^{\prime} / G\right)^{-3} \\
& u^{\prime \prime \prime}(\xi)=-6 A_{1}\left(G^{\prime} / G\right)^{4}-12 A_{1} \lambda\left(G^{\prime} / G\right)^{3}-A_{1}\left(7 \lambda^{2}+8 \mu\right)\left(G^{\prime} / G\right)^{2}-A_{1}\left(\lambda^{3}+8 \lambda \mu\right)\left(G^{\prime} / G\right) \\
& +B_{1}\left(\lambda^{2}+2 \mu\right)-A_{1}\left(\lambda^{2} \mu+2 \mu^{2}\right)+\left(8 B_{1} \lambda \mu+B_{1} \lambda^{3}\right)\left(G^{\prime} / G\right)^{-1}+B_{1}\left(7 \lambda^{2} \mu+8 \mu^{2}\right)\left(G^{\prime} / G\right)^{-2} \\
& +12 B_{1} \lambda \mu^{2}\left(G^{\prime} / G\right)^{-3}+6 B_{1} \mu^{3}\left(G^{\prime} / G\right)^{-4}
\end{aligned}
$$

Substitute (13) and (15) into (11) and after some simplifications we achieved an expression in $\left(G^{\prime} / G\right)^{i},(i=-4,-3,-2,-1,0,1,2,3,4)$.

Let the coefficient of $\left(G^{\prime} / G\right)^{i},(i=-4,-3,-2,-1,0,1,2,3,4)$ be zero, yields a set of algebraic equations about $A_{i}, B_{i}, w$ as follows:

Coefficient of $\left(\frac{G^{\prime}(\xi)}{G^{\prime}(\xi)}\right)^{-4}: 6 \alpha B_{1} \mu^{3}-3 / 2 w B_{1}^{2} \mu^{2}-6 \beta w^{2} B_{1} \mu^{3}=0$

Coefficient of $\left(\frac{G^{\prime}(\xi)}{G^{\prime}(\xi)}\right)^{-3}:-12 \beta w^{2} B_{1} \lambda \mu^{2}-3 w B_{1}^{2} \lambda \mu+12 \alpha B_{1} \lambda \mu^{2}=0$

Coefficient of $\left(\frac{G^{\prime}(\xi)}{G^{\prime}(\xi)}\right)^{-2}: \begin{aligned} & 3 w A_{1} B_{1} \mu^{2}+8 \alpha B_{1} \mu^{2}+w^{2} B_{1} \mu-B_{1} \mu-\frac{3}{2} w B_{1}^{2} \lambda^{2}-8 \beta w^{2} B_{1} \mu^{2} \\ & +7 \alpha B_{1} \lambda^{2} \mu-3 w B_{1}^{2} \mu-7 \beta w^{2} B_{1} \lambda^{2} \mu=0\end{aligned}$

Coefficient of $\left(\frac{G^{\prime}(\xi)}{G^{\prime}(\xi)}\right)^{-1}: \begin{aligned} & \alpha B_{1} \lambda^{3}+6 w A_{1} B_{1} \lambda \mu-w^{2} \beta B_{1} \lambda^{3}+8 \alpha B_{1} \lambda \mu-3 w B_{1}^{2} \lambda \\ & -B_{1} \lambda+w^{2} B_{1} \lambda-8 \beta w^{2} B_{1} \lambda \mu=0\end{aligned}$

Coefficient of $\left(\frac{G^{\prime}(\xi)}{G^{\prime}(\xi)}\right)^{0}: \begin{aligned} & -2 \alpha A_{1} \mu^{2}-\alpha A_{1} \lambda^{2} \mu+\alpha B_{1} \lambda^{2}-w^{2} A_{1} \mu+2 \beta w^{2} \mu^{2} A_{1}+w^{2} B_{1} \\ & -\beta w^{2} B_{1} \lambda^{2}+3 w \lambda^{2} A_{1} B_{1}-B_{1}+A_{1} \mu-3 / 2 w B_{1}^{2}+\beta w^{2} A_{1} \lambda^{2} \mu \\ & -2 \beta w^{2} \mu B_{1}+2 \alpha B_{1} \mu+6 w \mu A_{1} B_{1}-3 / 2 w A_{1}^{2} \mu^{2}=0\end{aligned}$ 
Coefficient of $\left(\frac{G^{\prime}(\xi)}{G^{\prime}(\xi)}\right): \begin{aligned} & -w^{2} \lambda A_{1}+8 \beta w^{2} A_{1} \lambda \mu+6 w \lambda A_{1} B_{1}-3 w A_{1}^{2} \lambda \mu \\ & +\beta w^{2} \lambda^{3} A_{1}-\alpha A_{1} \lambda^{3}-8 \alpha A_{1} \lambda \mu+A_{1} \lambda=0\end{aligned}$

Coefficient of $\left(\frac{G^{\prime}(\xi)}{G^{\prime}(\xi)}\right)^{2}: \begin{aligned} & -3 / 2 w A_{1}^{2} \lambda^{2}+7 \beta w^{2} A_{1} \lambda^{2}+8 \beta w^{2} A_{1} \mu-7 \alpha A_{1} \lambda^{2} \\ & -3 w A_{1}^{2} \mu-w^{2} A_{1}+3 w A_{1} B_{1}-8 \alpha \mu A_{1}+A_{1}=0\end{aligned}$

Coefficient of $\left(\frac{G^{\prime}(\xi)}{G^{\prime}(\xi)}\right)^{3}:-3 w A_{1}^{2} \lambda-12 \alpha A_{1} \lambda+12 \beta w^{2} A_{1} \lambda=0$

Coefficient of $\left(\frac{G^{\prime}(\xi)}{G^{\prime}(\xi)}\right)^{4}: 6 \beta w^{2} A_{1}-6 \alpha A_{1}-\frac{3}{2} w^{2} A_{1}^{2}=0$

Solving the above system of equations for $A_{1}, A_{0}, B_{1}$ by Maple 13, we achieve the following solutions:

Set 1: $A_{0}=$ const, $A_{1}=0, B_{1}= \pm \frac{4(\alpha-\beta) \mu}{\sqrt{\left\{\alpha\left(4 \mu-\lambda^{2}\right)+1\right\}\left\{\beta\left(4 \mu-\lambda^{2}\right)+1\right\}}}$ and

$$
w= \pm \sqrt{\frac{\alpha\left(4 \mu-\lambda^{2}\right)+1}{\beta\left(4 \mu-\lambda^{2}\right)+1}}
$$

Set 2: $A_{0}=$ const, $A_{1}= \pm \frac{4(\alpha-\beta)}{\sqrt{\left\{\alpha\left(4 \mu-\lambda^{2}\right)+1\right\}\left\{\beta\left(4 \mu-\lambda^{2}\right)+1\right\}}}, B_{1}=0$ and

$$
w= \pm \sqrt{\frac{\alpha\left(4 \mu-\lambda^{2}\right)+1}{\beta\left(4 \mu-\lambda^{2}\right)+1}}
$$

For the set 1 , we have the solutions

$$
u(\xi)=A_{0} \pm \frac{4(\alpha-\beta) \mu}{\sqrt{\left\{\alpha\left(4 \mu-\lambda^{2}\right)+1\right\}\left\{\beta\left(4 \mu-\lambda^{2}\right)+1\right\}}}\left(G^{\prime} / G\right)^{-1}
$$

When $\lambda^{2}-4 \mu>0$, then

$$
\begin{aligned}
& u_{ \pm}(\xi)=A_{0} \pm \frac{4(\alpha-\beta) \mu}{\sqrt{\left\{\alpha\left(4 \mu-\lambda^{2}\right)+1\right\}\left\{\beta\left(4 \mu-\lambda^{2}\right)+1\right\}}} \times \\
& \left(\frac{\sqrt{\lambda^{2}-4 \mu}}{2}\left(\frac{C_{1} \sinh \frac{1}{2}\left(\sqrt{\lambda^{2}-4 \mu} \xi\right)+C_{2} \cosh \frac{1}{2}\left(\sqrt{\lambda^{2}-4 \mu} \xi\right)}{C_{1} \cosh \frac{1}{2}\left(\sqrt{\lambda^{2}-4 \mu} \xi\right)+C_{2} \sinh \frac{1}{2}\left(\sqrt{\lambda^{2}-4 \mu} \xi\right)}\right)-\frac{\lambda}{2}\right)^{-1}
\end{aligned}
$$


where $\xi=x \mp \sqrt{\frac{\alpha\left(4 \mu-\lambda^{2}\right)+1}{\beta\left(4 \mu-\lambda^{2}\right)+1}} t$ and $A_{0}, C_{1}, C_{2}$ are arbitrary constants.

When $\lambda^{2}-4 \mu<0$, then

$$
\begin{aligned}
& u_{ \pm}(\xi)=A_{0} \pm \frac{4(\alpha-\beta) \mu}{\sqrt{\left\{\alpha\left(4 \mu-\lambda^{2}\right)+1\right\}\left\{\beta\left(4 \mu-\lambda^{2}\right)+1\right\}}} \\
& \left(\frac{\sqrt{4 \mu-\lambda^{2}}}{2}\left(\frac{-C_{1} \sin \frac{1}{2}\left(\sqrt{4 \mu-\lambda^{2}} \xi\right)+C_{2} \cos \frac{1}{2}\left(\sqrt{4 \mu-\lambda^{2}} \xi\right)}{C_{1} \cos \frac{1}{2}\left(\sqrt{4 \mu-\lambda^{2}} \xi\right)+C_{2} \sin \frac{1}{2}\left(\sqrt{4 \mu-\lambda^{2}} \xi\right)}\right)-\frac{\lambda}{2}\right)^{-1}
\end{aligned}
$$

where $\xi=x \mp \sqrt{\frac{\alpha\left(4 \mu-\lambda^{2}\right)+1}{\beta\left(4 \mu-\lambda^{2}\right)+1}} t$ and $A_{0}, C_{1}, C_{2}$ are arbitrary constants.

When $\lambda^{2}-4 \mu=0, \mu=\lambda=0$, then the solution is constant.

$$
u_{ \pm}(\xi)=A_{0}
$$

where $\xi=x \mp \sqrt{\frac{\alpha\left(4 \mu-\lambda^{2}\right)+1}{\beta\left(4 \mu-\lambda^{2}\right)+1}} t$ and $A_{0}, C_{1}, C_{2}$ are arbitrary constants.

For the set 2, we have the solutions

$$
u(\xi)=A_{0} \mp \frac{4(\alpha-\beta)}{\sqrt{\left\{\alpha\left(4 \mu-\lambda^{2}\right)+1\right\}\left\{\beta\left(4 \mu-\lambda^{2}\right)+1\right\}}}\left(G^{\prime} / G\right)
$$

When $\lambda^{2}-4 \mu>0$, then

$$
\begin{aligned}
& u_{\mp}(\xi)=A_{0} \mp \frac{4(\alpha-\beta)}{\sqrt{\left\{\alpha\left(4 \mu-\lambda^{2}\right)+1\right\}\left\{\beta\left(4 \mu-\lambda^{2}\right)+1\right\}}} \times \\
& \left(\frac{\sqrt{\lambda^{2}-4 \mu}}{2}\left(\frac{C_{1} \sinh \frac{1}{2}\left(\sqrt{\lambda^{2}-4 \mu} \xi\right)+C_{2} \cosh \frac{1}{2}\left(\sqrt{\lambda^{2}-4 \mu} \xi\right)}{C_{1} \cosh \frac{1}{2}\left(\sqrt{\lambda^{2}-4 \mu} \xi\right)+C_{2} \sinh \frac{1}{2}\left(\sqrt{\lambda^{2}-4 \mu} \xi\right)}\right)-\frac{\lambda}{2}\right)
\end{aligned}
$$

where $\xi=x \mp \sqrt{\frac{\alpha\left(4 \mu-\lambda^{2}\right)+1}{\beta\left(4 \mu-\lambda^{2}\right)+1}} t$ and $A_{0}, C_{1}, C_{2}$ are arbitrary constants.

When $\lambda^{2}-4 \mu<0$, then 


$$
\begin{aligned}
& u_{\mp}(\xi)=A_{0} \mp \frac{4(\alpha-\beta)}{\sqrt{\left\{\alpha\left(4 \mu-\lambda^{2}\right)+1\right\}\left\{\beta\left(4 \mu-\lambda^{2}\right)+1\right\}}} \\
& \left(\frac{\sqrt{4 \mu-\lambda^{2}}}{2}\left(\frac{-C_{1} \sin \frac{1}{2}\left(\sqrt{4 \mu-\lambda^{2}} \xi\right)+C_{2} \cos \frac{1}{2}\left(\sqrt{4 \mu-\lambda^{2}} \xi\right)}{C_{1} \cos \frac{1}{2}\left(\sqrt{4 \mu-\lambda^{2}} \xi\right)+C_{2} \sin \frac{1}{2}\left(\sqrt{4 \mu-\lambda^{2}} \xi\right)}\right)-\frac{\lambda}{2}\right)
\end{aligned}
$$

where $\xi=x \mp \sqrt{\frac{\alpha\left(4 \mu-\lambda^{2}\right)+1}{\beta\left(4 \mu-\lambda^{2}\right)+1}} t$ and $A_{0}, C_{1}, C_{2}$ are arbitrary constants.

When $\lambda^{2}-4 \mu=0, \mu=\lambda=0$, then

$$
u_{\mp}(\xi)=A_{0} \mp \frac{4(\alpha-\beta)}{\sqrt{\left\{\alpha\left(4 \mu-\lambda^{2}\right)+1\right\}\left\{\beta\left(4 \mu-\lambda^{2}\right)+1\right\}}} \times \frac{C_{2}}{C_{2} \xi+C_{1}}
$$

where $\xi=x \mp \sqrt{\frac{\alpha\left(4 \mu-\lambda^{2}\right)+1}{\beta\left(4 \mu-\lambda^{2}\right)+1}} t$ and $A_{0}, C_{1}, C_{2}$ are arbitrary constants.

Shape of solution (15) is similar to the shape of Figures of solutions (13).

Remark: All solutions presented in this paper have been checked with Maple by putting them back into the original equations.

\section{Explanation and Graphical Representation}

The solution $u(x, t)$ to the solitary wave Eq. (28), Eq. (32) and Eq. (34) play an important role for describing different types of wave propagation of pulses through optical fibers while shock waves appear in the supersonic jet flow. The Eq. (28), Eq. (32) and Eq.(34) are given not only more new multiple explicit solutions but also many types of exact traveling wave solutions. The exact traveling wave solutions are obtained from the explicit solutions by choosing the particular value of the physical parameters. So we can appropriate values of the parameters to obtained exact solutions. There are various types of traveling wave solutions that are particular interest in solitary wave theory. Wadati [28-30] defined soliton as a nonlinear wave characterized by the following properties.

(a) A localized wave propagates without change of its properties (shape, velocity etc.),

(b) Localized waves are stable against mutual collisions and retain their identities. This in turn means that soliton has the properties of particle. Soliton is a localized wave that has an infinite support or a localized wave with exponential tails.

In modern physics, a suffix-on is used to indicate the particle properties [28-30], for example phonon, photon etc. have particle properties. For this reason, the solitary wave with above 
properties of particle is called soliton. The soliton with two exponential tails from one asymptotic state to another is called kink solution but kink with singularities is called singular kink solutions. In this research work, some important traveling wave solutions are described and presented graphically.

The solution Eq.(28), Eq.(32) and Eq.(34) are presented the singular kink type soliton solution, are rise from one asymptotic state at to another asymptotic state. These solitons are referred to as topological solitons. The Fig. 1(a) (3D surface) and Fig. 1(b) (2D surface) of solution Eq. (28); the Fig. 3(a) (3D surface) and Fig. 3(b) (2D surface) of solution Eq. (32); the Fig. 5(a) (3D surface) and Fig. 5(b) (2D surface) of solution Eq. (34) has been shown in the following. Again in terms of sine-cosine function, we achieve the solutions Eq.(29) and Eq.(33) are periodic as like $\cos (x-t)$. The profile Fig.-2(a) (3D surface) and Fig-2(b) (2D surface) of solution Eq.(29); the profile Fig.4(a) (3D surface) and Fig-4(b) (2D surface) of solution Eq.(33) are shown in the below.

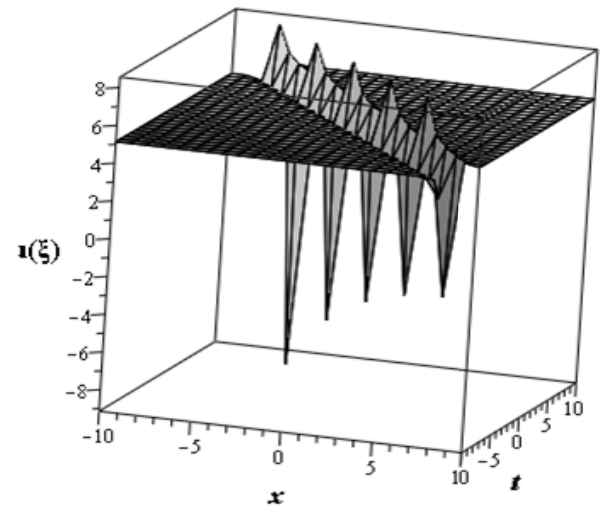

Fig 1(a). 3D Shape of Eq. (28) for $\lambda=3, \mu=1, \alpha=2, \beta=3, A_{0}=5, C_{2}=1, C_{2}=2$.

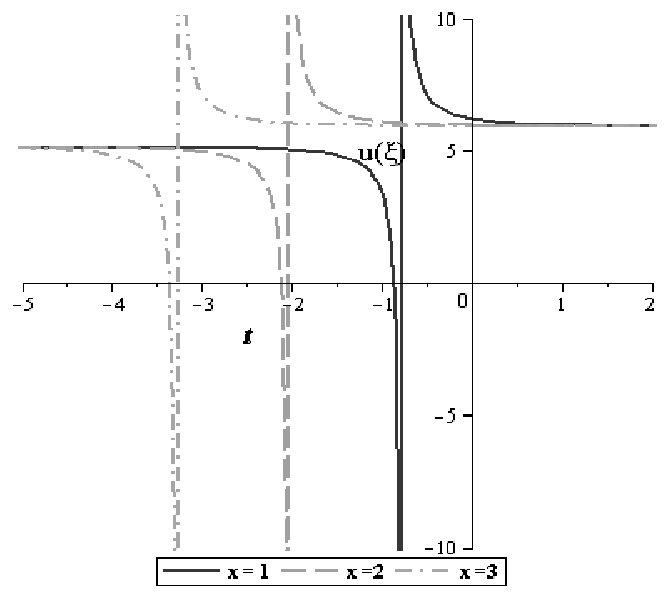

Fig 1(b). 2D Shape of Eq. (28) for the same values of the parameters of Fig 1(a). 


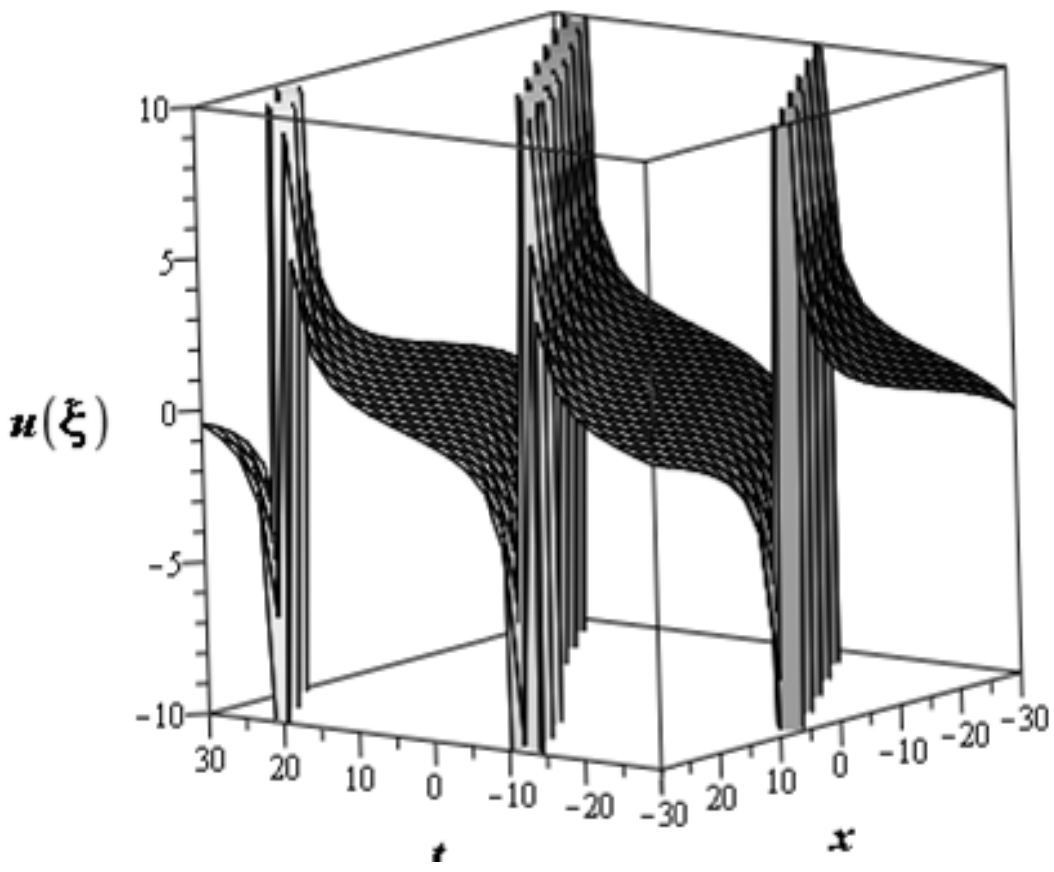

Fig 2(a). 3D Shape of Eq. (29) for $\lambda=1, \mu=2, \alpha=2, \beta=0.5, A_{0}=0.5, C_{2}=1, C_{2}=2$.

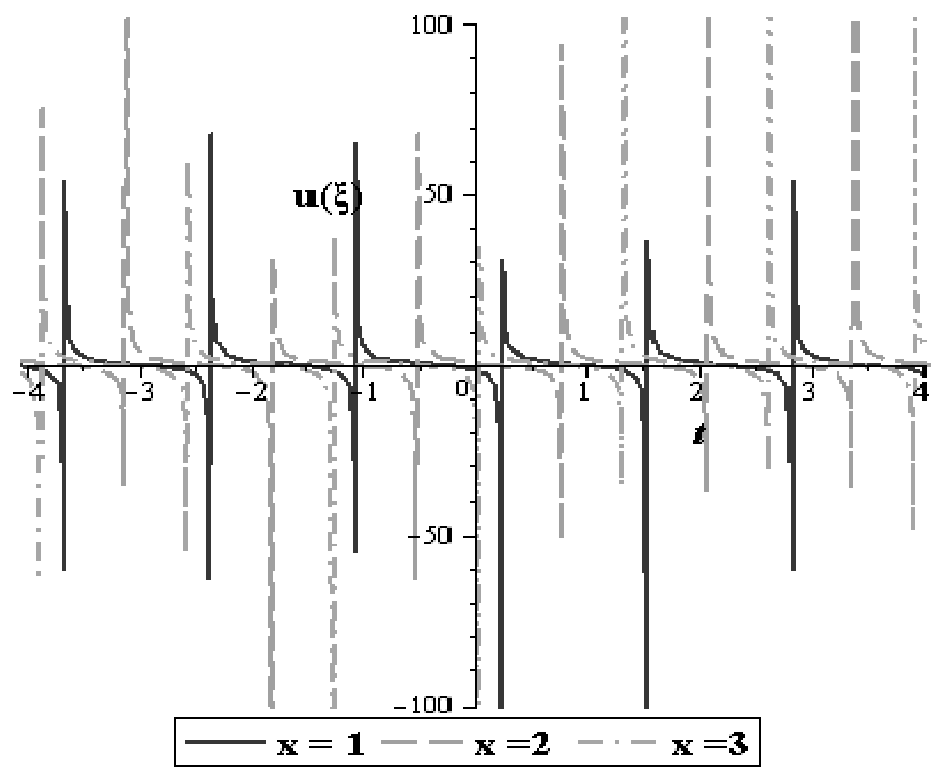

Fig 2(b). 2D Shape of Eq. (29) for the same values of the parameters of Fig 2(a). 


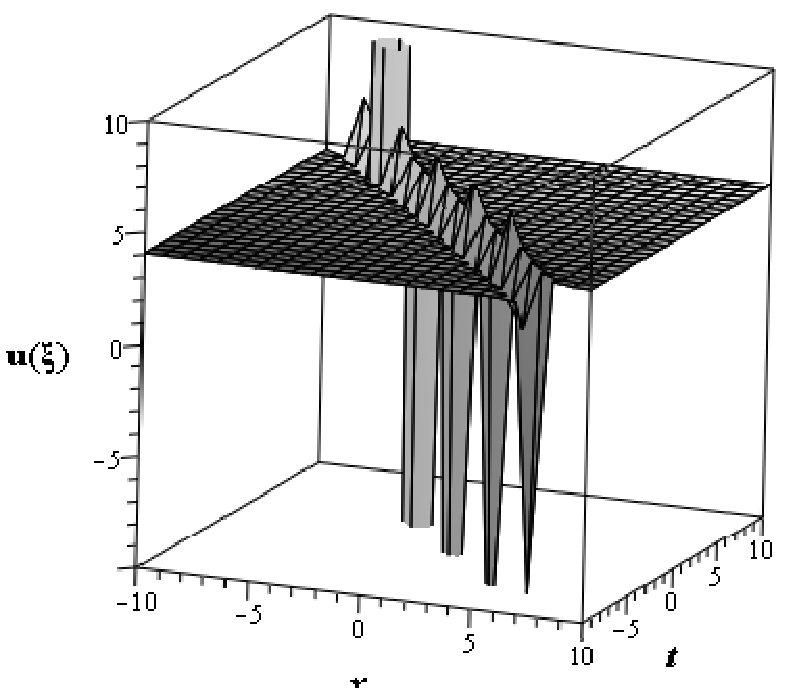

Fig 3(a). 3D Shape of Eq. (32) for $\lambda=3, \mu=1, \alpha=2, \beta=3, A_{0}=5, C_{2}=1, C_{2}=2$.

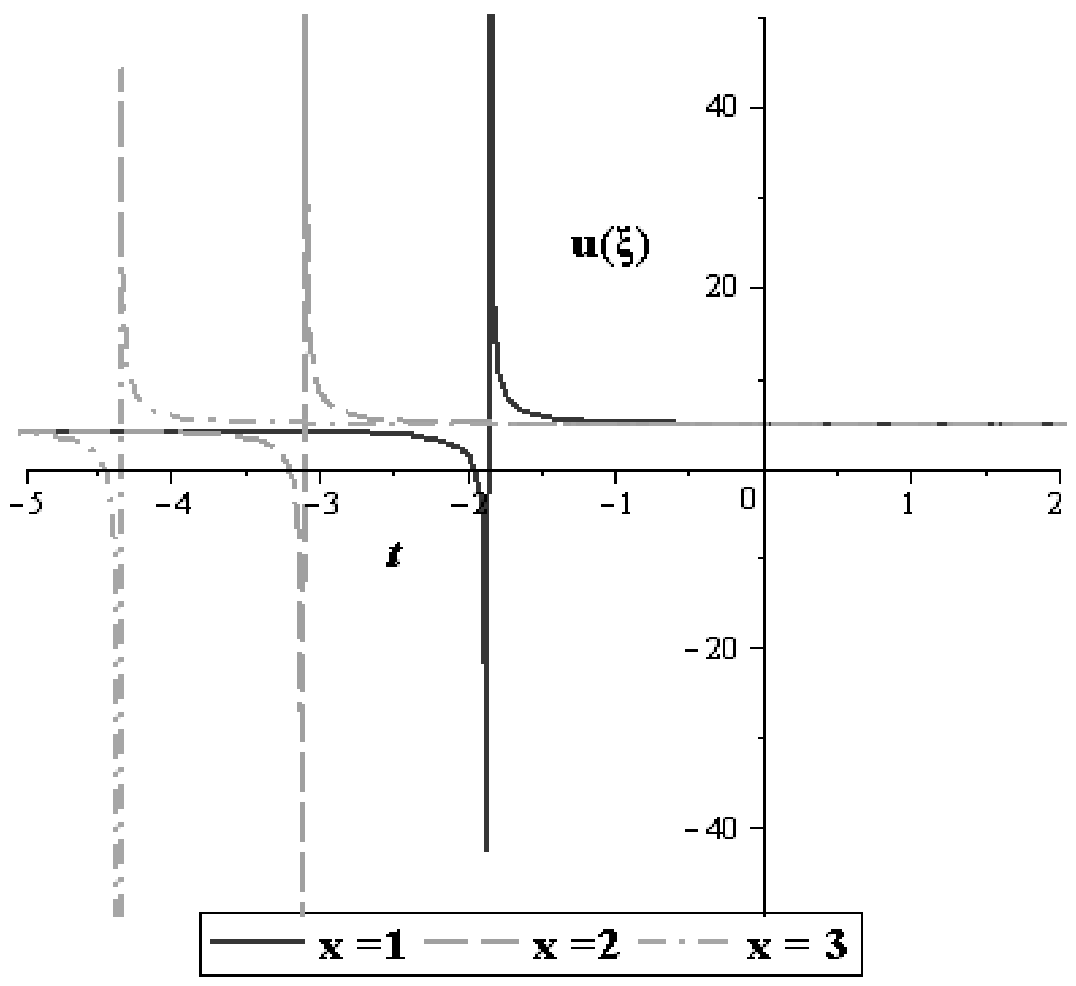

Fig 3(b). 2D Shape of Eq. (32) for the same values of the parameters of Fig 3(a). 


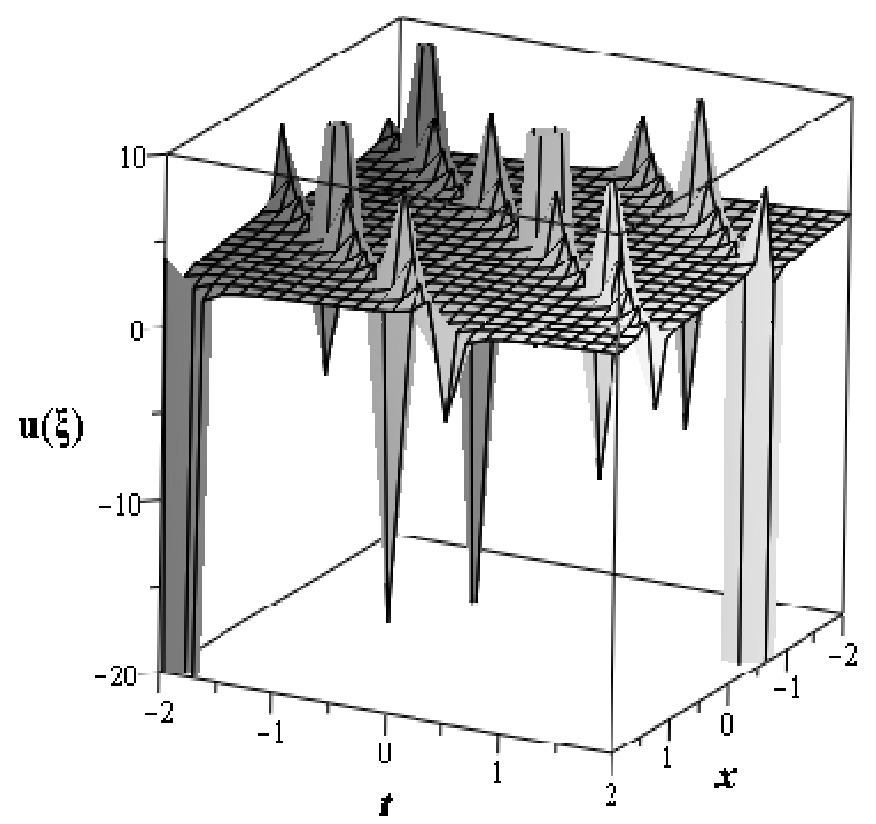

Fig 4(a). 3D Shape of Eq. (33) for $\lambda=1, \mu=3, \alpha=2, \beta=3, A_{0}=3, C_{2}=1, C_{2}=2$.

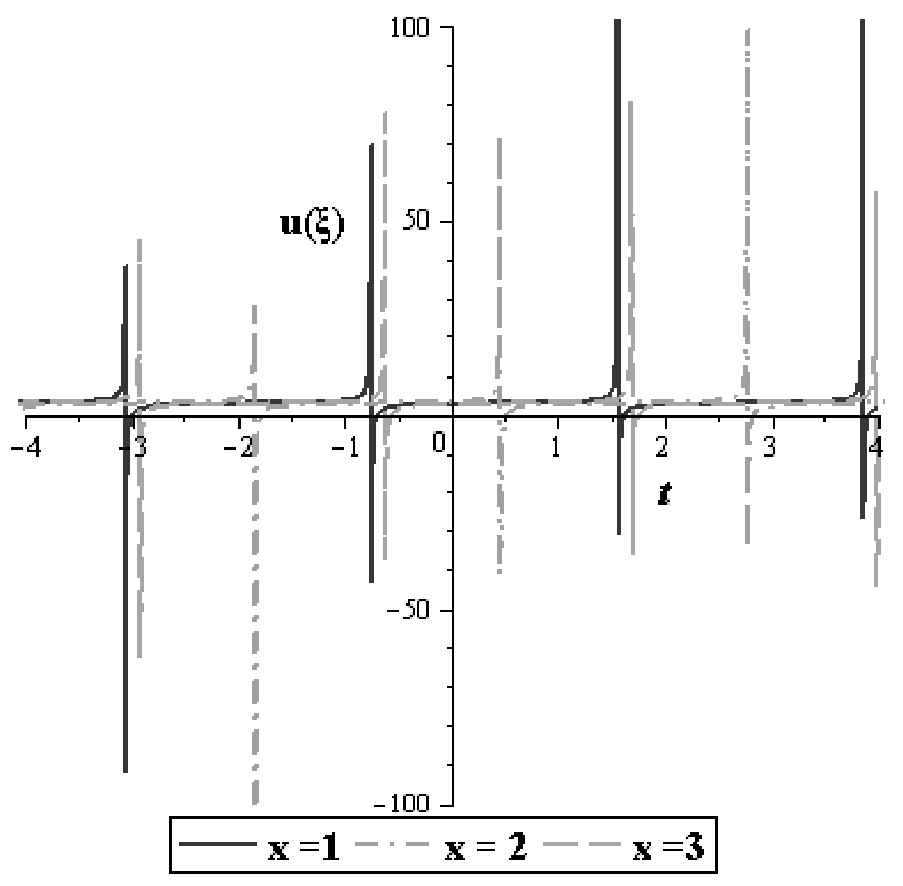

Fig 4(b). 2D Shape of Eq. (33) for the same values of the parameters of Fig 4(a). 


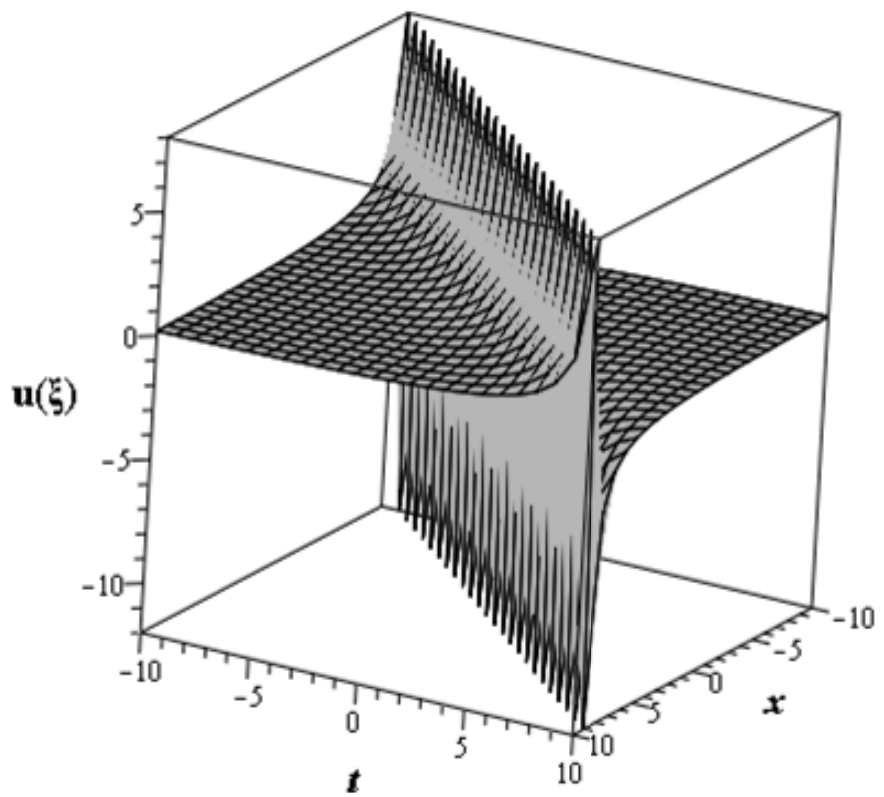

Fig 5(a). 3D Shape of Eq. (34) for $\lambda=\mu=0, \alpha=2, \beta=3, A_{0}=0, C_{2}=1, C_{2}=2$.

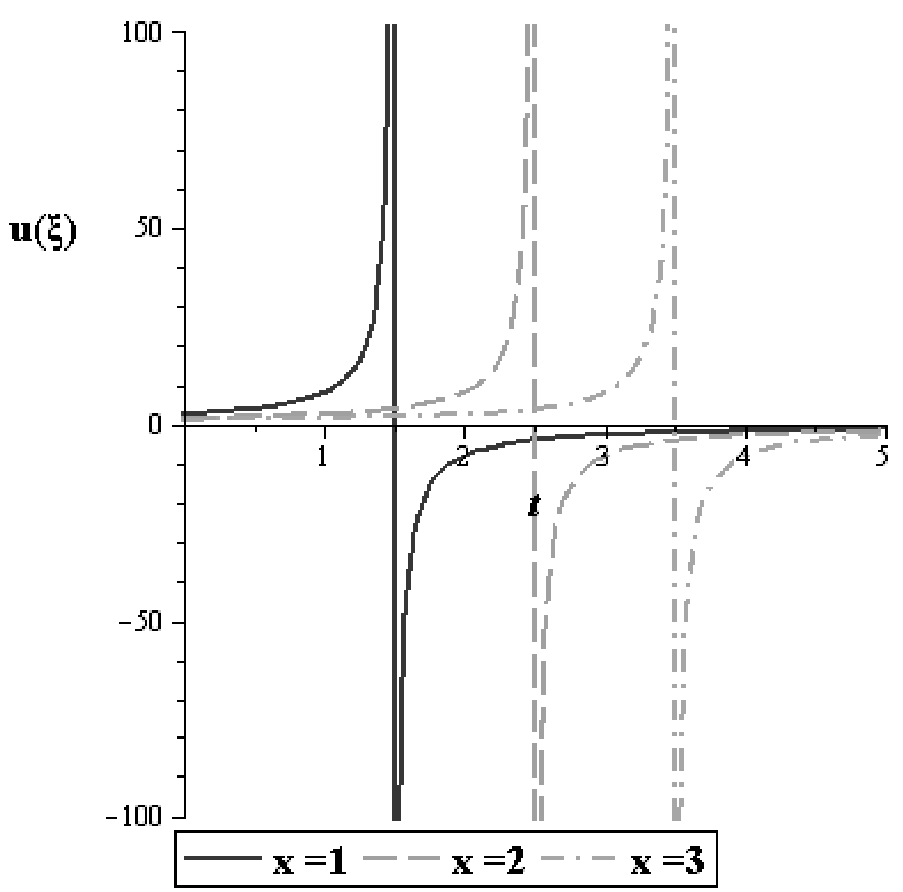

Fig 5(b). 2D Shape of Eq. (34) for the same values of the parameters of Fig 5(a). 


\section{Conclusions}

The improved $\left(G^{\prime} / G\right)$-expansion method is applied successfully to obtain exact traveling wave solutions of the Benney-Luke equation. As a result, we obtained more exact traveling wave solutions including trigonometric and hyperbolic solutions. We also presented 3D and 2D figures of the obtained solutions. Furthermore, the improved $\left(G^{\prime} / G\right)$ method appears to be quite creative, easier, faster and can be handled by computer easily and we used Maple-13 to solve the equation. The acquired solutions may be significant and important for analyzing the nonlinear phenomena arising in applied physical sciences. This will have a good sense to promote the extensive application of the equations.

\section{REFERENCES}

[1] R. W. Carroll and R. E. Showalter, Singular and Degenerate Cauchy Problems, vol. 12 of Mathematics in Science and Engineering, Academic Press, New York, NY, USA, 1976.

[2] S. L. Sobolev, Some new problems in mathematical physics, Izvestiya Akademii Nauk SSSR. Seriya Matematicheskaya, 18: 3-50, 1954.

[3] S. L. Sobolev, On a new problem of mathematical physics, Izvestiya Akademii Nauk SSSR. Seriya Matematicheskaya, 18: 3-50, 1954.

[4] S. A. Gabov, New Problems of the Mathematical Theory of Waves, Fizmatlit, Moscow, Russia, 1998.

[5] M. O. Korpusov, Yu. D. Pletner, and A. G. Sveshnikov, Unsteady waves in media with anisotropic dispersion, Zhurnal Vychislitel noi Matematiki i Matematicheskoi Fiziki, 39(6): pp. 1006-1022, 1999.

[6] M. O. Korpusov and A. G. Sveshnikov, Three-dimensional nonlinear evolution equations of pseudoparabolic type in problems of mathematical physics, Zhurnal Vychislitel'noi Matematiki I Matematicheskoi Fiziki, vol. 43, no. 12, pp. 1835-1869, 2003.

[7] P. I. Naumkin and I. A. Shishmarev, Nonlinear Nonlocal Equations in the Theory of Waves, vol. 133 of Translations of Mathematical Monographs, American Mathematical Society, Providence, RI, USA, 1994.

[8] A. T. Ali, New generalized Jacobi elliptic function rational expansion method. J. Comput Appl Math, 235 (2011) 4117-27.

[9] J. H. He, Application of homotopy perturbation method to nonlinear wave equations, Chaos, Solitons and Fractals. 26(3): (2005) 695-700.

[10] J. H. He, Variational principles for some nonlinear partial differential equations with variable coefficients, Chaos, Solitons and Fractals, 19 (4), (2004).

[11] G. Adomian, Solving Frontier Problems of Physics: The Decomposition Method, Kluwer Academic Publishers, Boston, 1994.

[12] K. Khan, M. A. Akbar, Exact and solitary wave solutions for the Tzitzeica-Dodd-Bullough and the modified KdV-Zakharov-Kuznetsov equations using the modified simple equation method, Ain Shams Engr. J., 4(4): 903-909, 2013.

[13] K. Khan, M. A. Akbar, Traveling wave solutions of the (2+1)-dimensional Zoomeron equation and the Burgers equations via the MSE method and the Exp-function method, Ain Shams Engr. J., 5(1): 247256, 2014.

[14] A. Bekir and A. Boz, Exact solutions for nonlinear evolution equation using Exp-function method, Physics Letters A 372(2008), 1619-1625.

[15] H. O. Roshid, N. Rahman and M.A. Akbar, Traveling waves solutions of nonlinear Klein Gordon equation by extended $\left(\mathrm{G}^{\prime} / \mathrm{G}\right)$-expasion method, Annals of Pure and Appl. Math. 2013, 3: 10-16. 
[16] Roshid, H. O., Uddin, M. H., Hossain, M. M. and Rahman, M. H. 2014 Exact traveling wave Solutions to Vakhnenko-Parkes equation, International journal of Software \& Hardware research in engineering, 2: $178-182$.

[17] Roshid, H.O., Akbar, M. A., Alam, M. N., Hoque, M. F. and Rahman, N. 2014 New extended (G'/G) expansion method to solve nonlinear evolution equation: the $(3+1)$-dimensional potential-YTSF equation, SpringerPlus, $3: 122$. doi:10.1186/2193-1801-3-122.

[18] Ma, W.X., Huang, T.W., Zhang, Y., A multiple exp-function method for nonlinear differential equations and its application. Physica Scripta 82 065003. 2010.

[19] Malfliet, W., Hereman, W., The tanh method: I. exact solutions of nonlinear evolution and wave equations. Physica Scripta 54 563-568, 1996.

[20] E. Fan, Extended tanh-method and its applications to nonlinear equations. Phy. Lett. A. 277, 212-218, 2000.

[22] Qawasmeh, A., 2013. Soliton solutions of (2+1)-dimensional Zoomeron equation, the Duffing equation and the SRLW equation. J. Math. Comput. Sci. 3: 1475-1480.

[23] Roshid, H.O., Kabir, M. R., Bhowmik, R.C., Datta, B. K., 2014. Investigation of Solitary wave solutions for Vakhnenko-Parkes equation via exp-function and $\operatorname{Exp}(-\varphi(\xi))$-expansion method. SpringerPlus 3: 692, 2014.

[24] Roshid, H.O., Rahman, M. A., 2014. The exp $(-\varphi(\eta))$-expansion method with application in the (1+1)dimensional classical Boussinesq equations. Results in Physics, 4: 150-155.

[25] S. Wang, G. Xu, and G. Chen, Cauchy problem for the generalized Benney-Luke equation, Journal of Mathematical Physics, vol. 48, no. 7, Article ID 073521, 2007.

[26] A. Gonz'alez N., The Cauchy problem for Benney-Luke and generalized Benney-Luke equations, Differential and Integral Equations, 20: 1341-1362, 2007.

[27] J. R. Quintero, A remark on the Cauchy problem for the generalized Benney-Luke equation, Differential and Integral Equations, 21: 859-890, 2008.

[28] J. R. Quintero, J. C. Munoz Grajales, Instability of solitary waves for a generalized Benney-Luke equation, Nonlinear Analysis, Theory, Methods and Applications, 68(4):. 3009-3033, 2008.

[29] M. Wadati, Introduction to solitons, Pramana: J. Physics 57(5-6): 841-847, 2001.

[30] M. Wadati, The exact solution of the modified Kortweg-de Vries equation, J. Phys. Soc. Japan 32: $1681-1687,1972$.

[31] M. Wadati, The modified Kortweg-de Vries equation, J. Phys. Soc. Japan 34: 1289-1296, 1973.

[32] J. Akter and M. A. Akbar, Exact solutions to the Benney-Luke equation and the Phi-4 equations by using modified simple equation method, Results in Physics, 5: 125-130, 2015.

[33] O. F. Gozukizil and S. Akcagil, Travelling Wave Solutions to the Benney-Luke and the Higher-Order Improved Boussinesq Equations of Sobolev Type, Abstract and Applied Analysis, Volume 2012 (2012), Article ID 890574, 10 pages. DOI: 10.1155/2012/890574. 